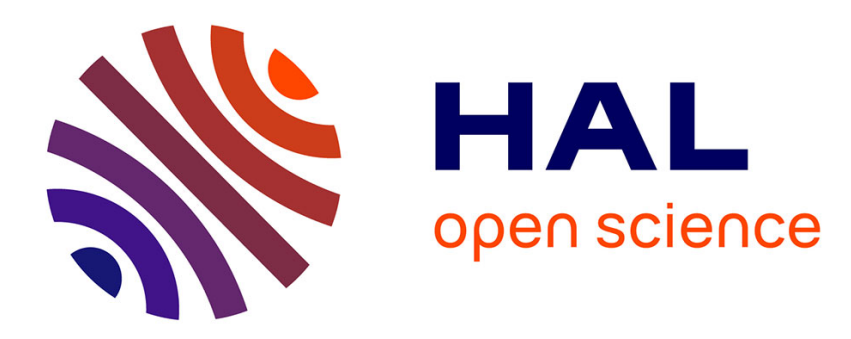

\title{
Statistics of the performance of time reversal in a lossy reverberating medium
}

\author{
Andrea Cozza
}

\section{To cite this version:}

Andrea Cozza. Statistics of the performance of time reversal in a lossy reverberating medium. Physical Review E: Statistical, Nonlinear, and Soft Matter Physics, 2009, 80 (5), pp.056604-056614. hal00444485

\section{HAL Id: hal-00444485 \\ https://hal.science/hal-00444485}

Submitted on 8 Nov 2010

HAL is a multi-disciplinary open access archive for the deposit and dissemination of scientific research documents, whether they are published or not. The documents may come from teaching and research institutions in France or abroad, or from public or private research centers.
L'archive ouverte pluridisciplinaire HAL, est destinée au dépôt et à la diffusion de documents scientifiques de niveau recherche, publiés ou non, émanant des établissements d'enseignement et de recherche français ou étrangers, des laboratoires publics ou privés. 


\title{
Statistics of the Performance of Time Reversal in a Lossy Reverberating Medium
}

\author{
Andrea Cozza* \\ SUPELEC, Département de Recherche en Électromagnétisme, 3 rue Joliot-Curie, 91192 Gif-sur-Yvette, France.
}

(Dated: October 20, 2009)

\begin{abstract}
It has been proposed [J. Derosny, Ph.D. Thesis, Université Paris VI, 2000] that the performance of time reversal at recreating a coherent pulse in a strongly reverberating medium is directly proportional to the number of resonant modes $M$ actively taking part at the transmission of energy. This idea is here tested against experimental results, showing that as soon as losses are taken into account, the quality of the focused pulse is a sublinear function of $M$, leading to a saturation phenomenon that was previously unacknowledged. This is here proven to be caused by mutual coupling between lossy resonant modes, thanks to a statistical modal description of the transmission of signals through the medium. Closed-form relationships are proposed for the first two moments of the pulse signal-to-noise ratio, linking them to the occupied bandwidth, the number of active modes and the degree of resonance of the medium. These formulae, supported by experimental and numerical results, prove that the performance of time reversal can be affected by a strong statistical dispersion. The proposed analysis also predicts that time reversal is a self-averaging process when applied to a reverberating medium, thus allowing the use of models developed in an ensemble-average framework.
\end{abstract}

\section{INTRODUCTION}

The interest of time-reversal techniques has been demonstrated in the fields of acoustics as well as electromagnetics, giving rise to a host of applications as diverse as pulsed-energy focusing in complex media [1], imaging techniques [2], and selective focusing [3]. Among these, one of the most surprising features of time reversal is its ability to ensure the transmission of coherent pulses through reverberating media $[1,4]$. In the context of this paper, we will consider a reverberating medium as a generally inhomogeneous medium where the propagation of electromagnetic or acoustic energy is strongly constrained into a finite volume. This region of space can be identified by an ideally closed surface imposing highly reflective boundary conditions, a configuration often referred to as a cavity. The provision of a finite volume does not exclude the existence of small apertures, through which a limited amount

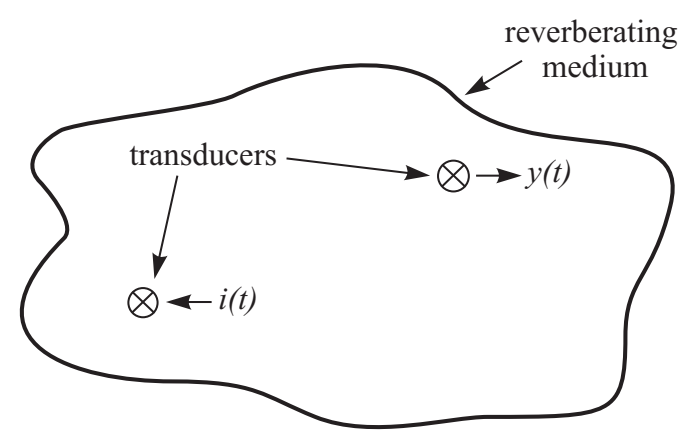

FIG. 1: Synoptic of a generic communication system embedded into a reverberating environment. A pulse $y(t)$ is to be focused at the receiver location by feeding the transmitter with an ad hoc signal $i(t)$ defined by means of time-reversal techniques.

*andrea.cozza@supelec.fr of the energy can leak out of the reverberating environment leading to a small perturbation of its behaviour assimilable to an energy loss. Coherently with this scenario, the media filling the cavity, as well as its boundary surface, will be regarded as lossy, introducing power dissipation along wave propagation. Following this description, a cavity is characterized by an infinite but countable set of resonances, associated to the eigenmodes of Helmholtz equation when the reflectivesurface boundary conditions are enforced [5].

Typically, for a pulse transmitted within such a medium, the signal received would be dominated by a long non-coherent tail, made up of a large number of echoes of the original pulse; conversely, use of time-reversal techniques allows a predominantly coherent transmission of the pulse. Applications of this property to electromagnetics range from new signal-forming schemes for telecommunications in multipath channels [6] to the generation of high-intensity local fields for device/material testing [7]. Although the basics for the physical interpretation of time-reversal in such context are known [4], there is no available model allowing to predict the statistical behaviour of this technique when used in a reverberating medium, and in particular how its performance depends on the relative position of the receiver-transmitter pair within the system (see Fig. 1). In particular, the analyses found in the literature focus just on the mean asymptotic performance, without giving any hint of its statistical dispersion. Furthermore, to the best of our knowledge, these models assume the system to be lossless [4].

In this paper we fill this gap by proposing a study of the performance of time reversal for more realistic scenarios, assessing how the signal-to-noise ratio (SNR) of received pulses evolves while changing, on the one hand, the positions of the transducers and, on the other hand, the properties of the medium. This is done by studying the statistics of the performance, in particular by proposing closed-form expressions for the first two moments of the SNR of the received pulse. These results should allow predicting more thoroughly the way time reversal behaves in a reverberating medium, especially thanks to the knowledge of the variance of the SNR: indeed, this is a fundamental piece of data for ensuring, within a certain confidence margin, a given performance for any po- 
sition of the receiver and transmitter. Moreover, by acknowledging the existence of non-negligible loss mechanisms, the proposed model predicts phenomena that were not previously highlighted, such as the fact that losses lead to a saturation of the SNR, because of the mutual coupling between resonant modes through the tails of their frequency spectra. A major point is the generality of these results, which are valid for any system obeying to a modal resonant description, be it acoustic or electromagnetic, while requiring a very limited number of simplifying assumptions.

The paper starts with the introduction of tools for assessing the quality of the received pulse, generalized to the case of lossy media by applying novel definitions with respect to [4]. These tools are then applied for the analysis of experimental results obtained in an electromagnetic reverberation chamber, pointing out some of the previously recalled phenomena that have not yet been acknowledged. A theoretical analysis based on a modal description is then proposed in Section III, leading to the first two statistical moments of the SNR; subsequently, we focus in Section IV on the asymptotic response, proving that thanks to the self-averaging properties of time reversal, its statistical description is asymptotically independent on the actual realization of the reverberating medium, and entirely describable through few global parameters. Numerical results, as well as experimental ones are presented in Section V, validating the accuracy of the proposed analysis. Finally, a simple heuristic interpretation of our findings is given in Section VI, providing a framework for intuitively understanding the reasons for the saturation of the SNR.

\section{ON THE QUALITY OF RECEIVED PULSES}

\section{A. Mathematical tools for quality assessment}

In this Section we are concerned by the use of time-reversal techniques for transmitting a coherent pulse to a given receiver placed in a reverberating medium. Hereafter, this will be supposed to be reciprocal. The configuration we deal with is depicted in Fig. 1: two transducers are placed within the medium, one acting as a transmitter and the other one as a receiver. Defining $h(t)$ as the impulse response between the two transducers, it was shown in [4] that by transmitting the signal $i(t)=x(-t) \star h(-t)$, the received signal $y(t)$ will be a fair replica of $x(-t)$, even in a strongly reverberating medium. Due to this feature, time reversal has been proposed as a way of communicating through complex media, and in particular multipath channels, whose characteristics are well represented by reverberating media [8]. An example of pulse received for this setup is shown in Fig. 2, where $x(t)$ is a cardinal sinus modulating an harmonic carrier. Indeed, it appears that the received signal is almost undistinguishable from the original one around its peak region, whereas it is affected by a stronger modification over the signal tails.

In the following, we will consider the pulse $x(t)$ to have a spectral content comprised in the frequency range $\left[f_{1}, f_{2}\right]$, i.e., with a frequency bandwidth $B_{T}=f_{2}-f_{1}$ centered around the frequency $f_{c}=\left(f_{1}+f_{2}\right) / 2$. A total number of $M$ reso-
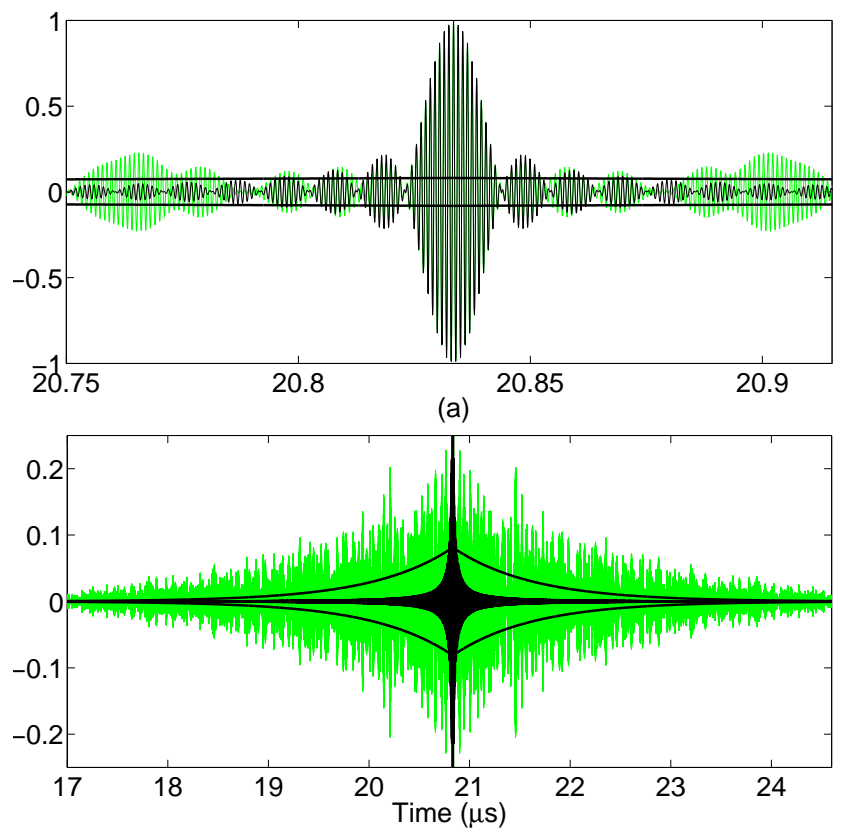

(b)

FIG. 2: An example of a pulse transmitted through a reverberating medium using time reversal, for the case of $Q=5000, M=500$, $B_{T}=100 \mathrm{MHz}$, central frequency $1 \mathrm{GHz}$ : the region around the peak of the pulse (a) and a wider perspective highlighting the residual noise distribution (b). The dark trace is the original pulse to be transmitted, whereas the light one is the signal actually received, affected by residual noise. The thicker line represents the equivalent noise $n_{e}(t)$.

nant modes will be assumed to exist over this frequency range, with resonance frequencies given by the set $\left\{f_{k}\right\}$. In a general way, a transfer function $H(f)$ in a reverberating medium can be expressed as a superposition of these modes, weighted by complex coefficients $\gamma_{k}=\alpha_{k}+\mathrm{j} \beta_{k}$ [5], i.e.,

$$
H(f)=\sum_{k=1}^{M} \gamma_{k}(f) \phi_{k}(f) \quad, \quad f \in\left[f_{1}, f_{2}\right] .
$$

The response $\phi_{k}(f)$ of the $k$-th mode will be assumed, with no loss of generality, to be a Lorentzian function. By considering the main effect of losses to lead to a small perturbation of these functions, one can write [5]:

$$
\phi_{k}(f)=\frac{f_{k}^{2} / Q_{k}}{f_{k}^{2}\left(1+\mathrm{j} / 2 Q_{k}\right)^{2}-f^{2}},
$$

where $Q_{k}$ is the quality factor associated to the $k$-th mode, which thus has a $-3 \mathrm{~dB}$ bandwidth $B_{M, k}=f_{k} / Q_{k}$. The modal weights $\left\{\gamma_{k}\right\}$ are functions of the transmitter and receiver positions and of the spatial field distribution associated to each resonant mode.

In order to simplify the notations in the following analysis, we will consider the reference signal to be $x(-t)$, so that the received one is rather linked to $x(t)$. For the same reason, we 
will avoid delaying the time-reversed pulse, and consider a non-causal description, as this does not affect the final results. Bearing these definitions in mind, the fitness of the focused pulse can be assessed by computing the component $s(t)=$ $\rho x(t)$ that is coherent with respect to $x(t)$, leading to

$$
y(t)=\rho x(t)+n(t),
$$

where $n(t)$ is the residual noise due to the distortion of the pulse introduced by the non-flat transfer function $H(f)$ of the medium, with $H(f)$ the Fourier transform of $h(t)$. Residual $n(t)$ being orthogonal to $x(t)$ by definition, $\rho$ can be computed as

$$
\rho=\frac{\langle Y, X\rangle}{\langle X, X\rangle}=\frac{\int_{f_{1}}^{f_{2}} X|H|^{2} X^{\star} \mathrm{d} f}{\int_{f_{1}}^{f_{2}}|X|^{2} \mathrm{~d} f},
$$

having applied Parseval equality, where $X(f)$ and $Y(f)$ are the Fourier spectra of the respective time-domain signals. The brackets stand for the projection operator. It is interesting to notice that because of the quadratic form in the previous result, $\rho \in \mathbb{R}^{+}$, so that the sign of the peak of the received pulse will always be unchanged. Defining the energy $\mathcal{E}_{G}$ of a spectrum $G(f)$ as

$$
\mathcal{E}_{G}=2 \int_{f_{1}}^{f_{2}}|G(f)|^{2} \mathrm{~d} f \quad,
$$

the energy $\mathcal{E}_{S}$ of the coherent part is thus given by

$$
\mathcal{E}_{S}=\rho^{2} \mathcal{E}_{X}=2 \rho^{2} \int_{f_{1}}^{f_{2}}|X|^{2} \mathrm{~d} f,
$$

whereas the energy $\mathcal{E}_{N}$ of the residual noise is

$$
\mathcal{E}_{N}=2 \int_{f_{1}}^{f_{2}}|X|^{2}|H|^{4} \mathrm{~d} f-\mathcal{E}_{S},
$$

so that we can introduce the energy $\operatorname{SNR} \Lambda$ as

$$
\Lambda=\frac{\mathcal{E}_{S}}{\mathcal{E}_{N}} .
$$

While the energy ratio $\Lambda$ will be extensively used in the rest of the paper, the ability of time reversal in transmitting coherent pulses is better assessed by means of the peak SNR $\Lambda_{p}$ as defined as follows [4]:

$$
\Lambda_{p}=\frac{s^{2}(0)}{n_{\mathrm{rms}}^{2}}
$$

having assumed that the peak of the received pulse occurs in $t=0$. The quantity $n_{\mathrm{rms}}$ is the root-mean-square (rms) value of $n(t)$ :

$$
n_{\mathrm{rms}}^{2}=\lim _{T \rightarrow \infty} \frac{1}{T} \int_{-T / 2}^{T / 2} n^{2}(t) \mathrm{d} t
$$

Equation (9) thus measures how much the coherent part of the transmitted pulse stands out of the residual noise. Now, the use of the rms value is consistent only in the case of a lossless system, as done in [4], since in this case $n(t)$ would have an infinite energy but a finite non-zero average power. Conversely, for the case of a lossy system, $n_{\mathrm{rms}}$ would be equal to zero, since the noise has finite energy; as a matter of fact, the time-constant characterizing the decay of a mode with quality factor $Q_{k}$ is $\tau_{k}=Q_{k} /\left(\pi f_{k}\right)=1 /\left(\pi B_{M, k}\right)$. We will assume that all the modes involved have the same bandwidth, and as a consequence the same time-constant $\tau=\tau_{k}, \forall k$; this assumption is valid as long as $B_{T} / f_{c}$ is sufficiently smaller than one, i.e., for configurations that cannot yet be regarded as wide-band, though not strictly narrow-band. Under such conditions, $n(t)$ will also obey to a time-decay with constant $\tau$; therefore, we introduce the equivalent noise signal $n_{e}(t)$ :

$$
n_{e}(t)=n_{0} \mathrm{e}^{-\pi f_{c} t / Q},
$$

where $Q$ is the average quality factor, and by imposing the same overall energy for the two noise signals, we get

$$
n_{0}^{2}=\frac{\mathcal{E}_{N} \pi f_{c}}{Q}
$$

The equivalent noise $n_{e}(t)$ behaves as a smoothed version of the actual noise $n(t)$, maintaining the same overall timedecay, and thus the same average instantaneous power content. The example shown in Fig. 2 illustrates this approach. It is now possible to define the peak SNR by considering the equivalent instantaneous noise energy at the signal peak in $t=0$,

$$
\Lambda_{p}=\frac{s^{2}(0)}{n_{e}^{2}(0)}=\frac{Q}{\pi f_{c}} \chi \Lambda
$$

where $\chi=x^{2}(0) / \mathcal{E}_{X}$ is a factor related to the shape of $x(t)$. This simple relationship between the two SNR definitions allows focusing on the energy SNR, which is much simpler to compute in the frequency domain.

Thanks to these definitions, it is possible to predict the performance of time-reversal transmission for any pulse $x(t)$, just by knowing the transfer function $H(f)$. Most remarkably, this just requires having access to the absolute values of the spectra $X(f)$ and $H(f)$.

\section{B. Experimental investigations in a reverberation chamber}

In general, the pulse SNR will depend on the relative position of the transmitter and the receiver within the system; in order to exploit the time-reversal technique for real-life applications, it is of paramount importance to be able to ensure that a given minimum SNR be respected for any transducers position, at least with respect to a certain confidence margin. In order to assess the variability of the SNR, we carried out experimental tests, by considering an electromagnetic reverberation chamber, with a fixed antenna acting as the transmitter and a linearly polarized electro-optical sensor (connected to 
an optical fiber) as a receiver. This last choice was imposed by the fact that a receiving antenna being moved inside the chamber would have changed its fundamental characteristics, modifying the sets $\left\{f_{k}\right\}$ and $\left\{\gamma_{k}\right\}$, and as thus impairing the validity of any comparison. The very weak interaction ensured by the optical sensor means that it can be regarded as an almost ideal electric-field probe, minimizing the modification of the quantity being measured.

A total of 100 randomly chosen positions and orientations of the receiver were considered, measuring the respective transfer functions over six frequency ranges, centered at frequencies from $0.5 \mathrm{GHz}$ to $3 \mathrm{GHz}$, by steps of $0.5 \mathrm{GHz}$. For each central frequency, two bandwidths $B_{T}$ were considered, namely $100 \mathrm{MHz}$ and $200 \mathrm{MHz}$.

We assumed, for the sake of simplicity, $x(t)$ to have a flat spectrum over the frequency range $\left[f_{1}, f_{2}\right]$; this would be the case, e.g., for cardinal sinus pulses. The energy SNR was computed by means of (8), leading to the empirical statistical distributions shown in Fig. 3, and the first two statistical moments of $\Lambda$ resumed in Table I, together with the average peak SNR $\Lambda_{p}$. These results point to three important facts: 1 ) while the average energy SNR increases with the frequency, its progression slows down at the same time, converging on an asymptotic value close to one, 2) the actual value of $\Lambda$ is strongly dispersed, depending on the position of the receiver and, 3) an increasing bandwidth has apparently little effect on the average energy SNR, whereas its dispersion decreases. To the best of our knowledge, these conclusions have never been discussed before, and no theoretical framework is available for interpreting them.

The only available model is the one proposed in [4] for a lossless configuration. It predicts a direct proportionality between the number of modes $M$ and the average peak SNR $\Lambda_{p}$. In order to apply this model to our experimental results, we estimated the number $M_{\mathrm{w}}$ of resonant modes existing in the chamber for a given frequency range through Weyl's formula [9]

$$
M_{\mathrm{w}}\left(f_{c}\right) \simeq \frac{8 \pi V}{c^{3}} f_{c}^{2} B_{T}\left(1+\frac{B_{T}^{2}}{12 f_{c}^{2}}\right),
$$

where $V$ is the volume of the reverberation chamber and $c$ is the speed of light in the medium filling it. Results obtained for the case of Supelec's chamber $\left(V=3.08 \times 1.84 \times 2.44 \mathrm{~m}^{3}\right)$ are shown in Table I. By comparing $M_{\mathrm{w}}$ to the average $\Lambda_{p}$, it is clear that their relationship is more complex, and characterized by a form of saturation of the performance, since even for large increases in $M_{\mathrm{w}}, \Lambda_{p}$ is barely affected.

The reasons for such a peculiar behaviour are to be sought in the existence of loss mechanisms in actual reverberation chambers; we will show in the next Section that this leads to mutual couplings between resonant modes, and ultimately to a saturation of the performance.

Concerning the statistical dispersion discussed in points 2) and 3), it has never been addressed before. It is worthwhile noticing that the orientation of the probe has little effect on the dispersion, since the cavity was over-moded for all the frequency ranges, apart for $f_{c}=0.5 \mathrm{GHz}$. For such configurations, the field is statistically isotropic, and it presents very

\begin{tabular}{ccccccc}
\hline \hline$f_{c}(\mathrm{GHz})$ & $B_{T}(\mathrm{MHz})$ & mean $\Lambda$ & $\operatorname{std} \Lambda$ & mean $\Lambda_{p}$ & $Q$ & $M_{\mathrm{w}}$ \\
\hline \multirow{2}{*}{0.5} & 100 & 0.37 & 0.074 & 187 & 3900 & 322 \\
& 200 & 0.30 & 0.051 & 300 & & 644 \\
\multirow{5}{*}{1.0} & 100 & 0.75 & 0.12 & 272 & 5700 & 1287 \\
& 200 & 0.72 & 0.080 & 130 & & 2574 \\
\multirow{4}{*}{1.5} & 100 & 0.91 & 0.14 & 243 & 6300 & 2896 \\
& 200 & 0.90 & 0.10 & 481 & & 5792 \\
2.0 & 100 & 0.96 & 0.13 & 192 & 6300 & 5149 \\
& 200 & 0.95 & 0.12 & 380 & & 10297 \\
2.5 & 100 & 1.02 & 0.13 & 187 & 7200 & 8045 \\
& 200 & 1.00 & 0.10 & 365 & & 16090 \\
3.0 & 100 & 1.02 & 0.14 & 188 & 8700 & 11584 \\
& 200 & 0.98 & 0.10 & 358 & & 23169 \\
\hline \hline
\end{tabular}

TABLE I: Statistical moments of the energy SNR $\Lambda$ and average peak SNR $\Lambda_{p}$, as computed from the experimental data presented in Fig. 3. The approximate number of modes $M_{\mathrm{w}}$ was computed by means of Weyl's formula (14), whereas the average quality factor $Q$ was directly estimated from the time constant of the residual noise $n(t)$.

similar statistical properties along its three Cartesian components [10].

These results point out that apart from being able to explain the limitations of time reversal, it is of paramount importance to have a model predicting the statistical dispersion of the SNR. These are indeed the basic motivations of this paper.

\section{STATISTICAL MOMENTS OF $\Lambda$}

As often done in statistical descriptions, the parameters of the model will need to be regarded as random variables. This approach is not just dictated by mathematics, but it comes with physical meaning. In particular, the resonance frequencies $\left\{f_{k}\right\}$ are indeed distributed over the bandwidth $B_{T}$ in a way that is hardly predictable, unless in canonical configurations. For the associated modal weights $\left\{\gamma_{k}\right\}$, since describing the projection of the transducer characteristic response over the modal topographies, a modification in the position of the receiver or the transmitter leads to a modification of the $\left\{\gamma_{k}\right\}$, so that a random position of the transducers implies a random set of modal weights. Moreover, the fact that the modal topographies, as well the excitation of the transducers are, in general, sign-changing functions, implies that the $\left\{\alpha_{k}\right\}$ and $\left\{\beta_{k}\right\}$ should be treated as zero-mean random variables, and they will be assumed to be independent and identical distributed (iid). No further assumption will be necessary about the type of distributions.

In order to simplify the model, the $\phi_{k}(f)$ will be assumed to be frequency-shifted replica, with approximately the same bandwidth $B_{M}=f_{k} / Q_{k}, \forall k$. This also implies that all the modal responses $\phi_{k}(f)$ have the same energy $\mathcal{E}_{\phi}$. In principle, this assumption holds only when relatively narrow bands are considered, although the results shown in Section $\mathrm{V}$ prove that 


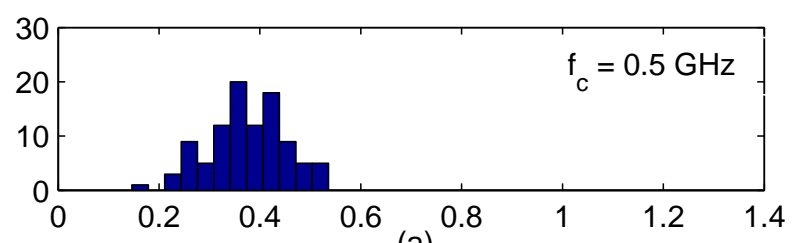

(a)

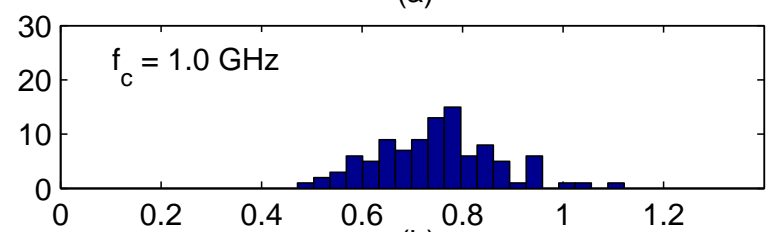

(b)
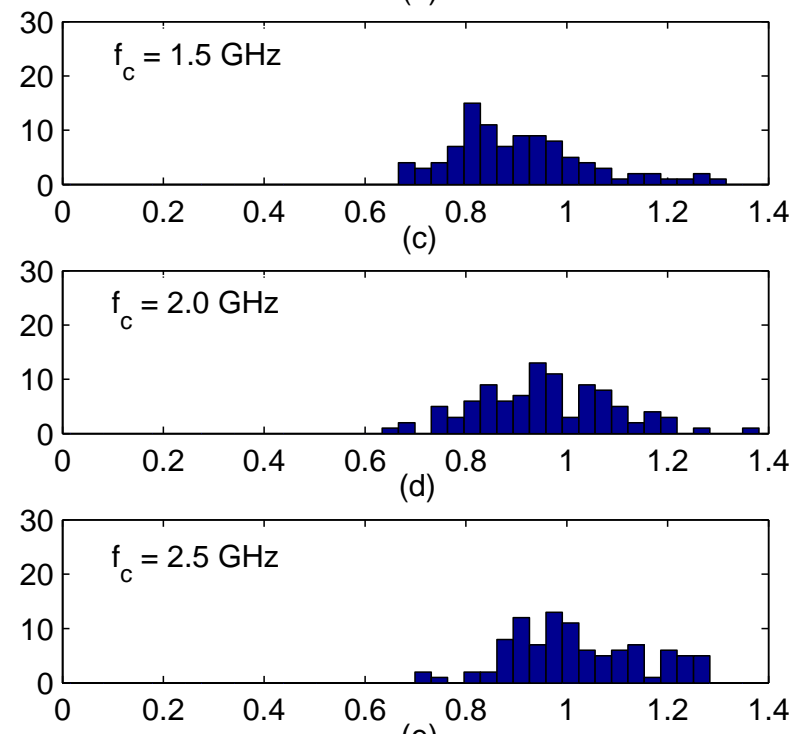

(e)

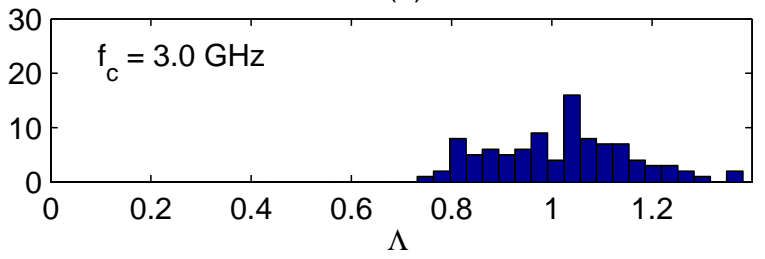

(f)
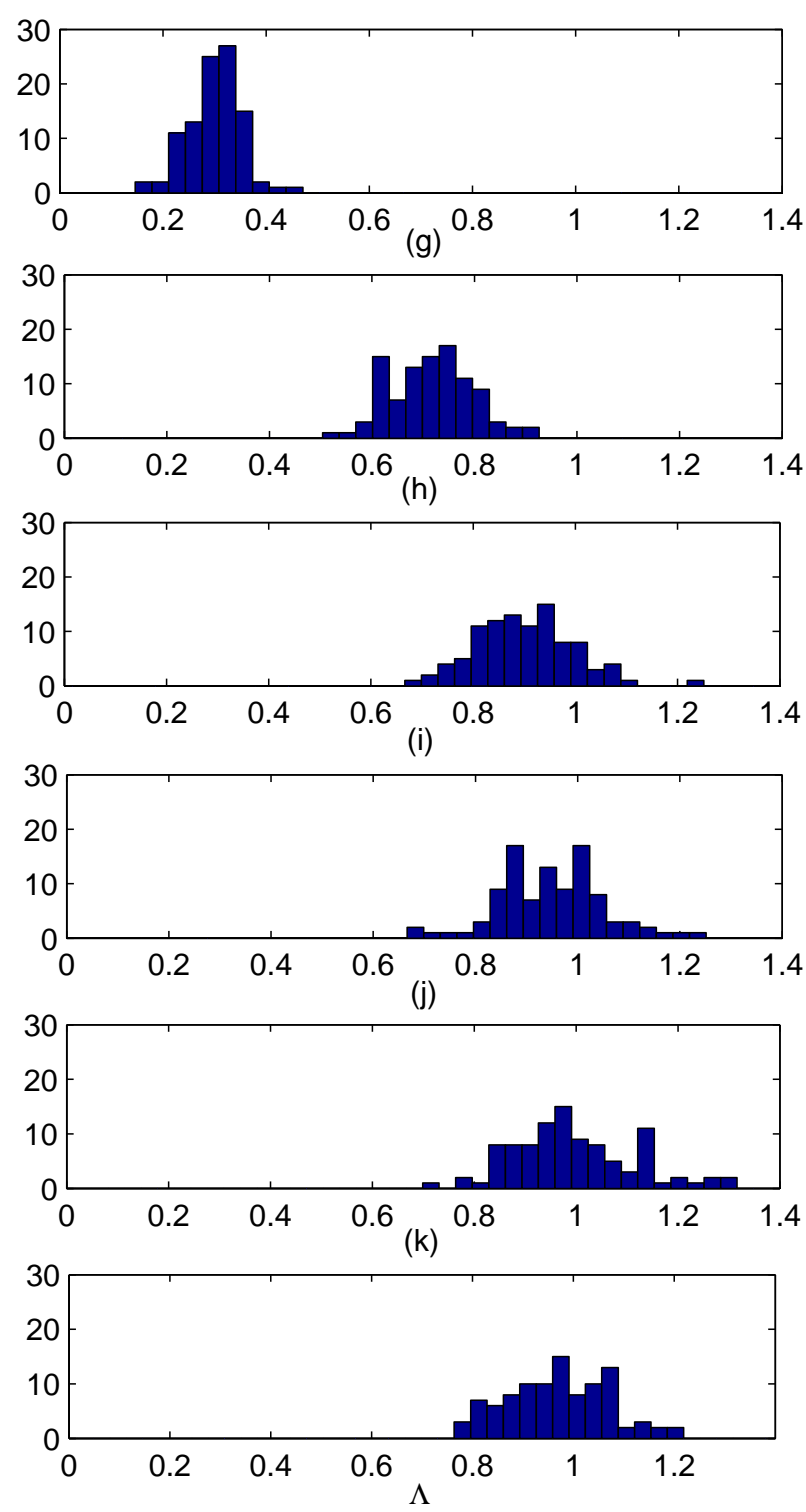

(I)

FIG. 3: Frequencies of occurrence for the energy SNR $\Lambda$ as computed from experimental data measured over a bandwidth $B_{T}=100 \mathrm{MHz}$ (left column) and $B_{T}=200 \mathrm{MHz}$ (right column), centered around the frequencies $f_{c}=\{0.5,1,1.5,2,2.5,3\} \mathrm{GHz}$ (top to bottom). Each histogram was obtained from a population of 100 sample transfer functions as measured between a fixed transmitter antenna and a moving electro-optical probe.

this is not necessarily the case.

It is important to understand the physical role of the following statistical analysis, which aims at accounting for the impact of the random position of the transducers on the received pulse SNR. Indeed, equation (1), when coupled with equations (6)-(8), leads to the definition of $\Lambda$ as a random function, depending on the probability density functions (pdfs) of $\left\{\gamma_{k}\right\}$ and $\left\{f_{k}\right\}$.

We start our analysis by considering a specific configuration for the reverberating medium, i.e., for a given set of known deterministic $\left\{f_{k}\right\}$, whereas the $\left\{\gamma_{k}\right\}$ will be regarded as random variables. This scenario corresponds to the case of a single realization for the medium, while the positions of the transducers are let free to change, so that all the statistical moments will be conditional to the set $\left\{f_{k}\right\}$. The ensemble behaviour of the SNR considering random $\left\{f_{k}\right\}$ will be studied in Section IV.

In order to simplify our analysis, but with no loss of generality in the conclusions, we will assume the modulus of $X(f)$ to be directly proportional to the characteristic function of the interval $\left[f_{1}, f_{2}\right]$, leading to $\chi=2 B_{T}$. This choice corresponds, e.g., to a cardinal sine excitation in the time-domain, modulating an harmonic carrier of frequency $f_{c}$, as for the example shown in Fig. 2. Attention should be paid to the fact that the definitions of the SNR actually depends just on the modulus of $X(f)$, so that an infinite number of pulse shapes 
sharing the same spectral occupation would be characterized by the same SNR.

Following this assumption, (8) can be recast as

$$
\Lambda=\frac{\left(\int_{f_{1}}^{f_{2}}|H|^{2} \mathrm{~d} f\right)^{2}}{B_{T} \int_{f_{1}}^{f_{2}}|H|^{4} \mathrm{~d} f-\left(\int_{f_{1}}^{f_{2}}|H|^{2} \mathrm{~d} f\right)^{2}} .
$$

Thus $\Lambda$ is entirely defined by the properties of the random function $|H(f)|^{2}$ over the frequency-range $\left[f_{1}, f_{2}\right]$. In order to study the statistical properties of $\Lambda$, we introduce the auxiliary random variables $W_{i} \in \mathbb{R}^{+}$, as defined as

$$
W_{i}=\int_{f_{1}}^{f_{2}}|H|^{2 i} \mathrm{~d} f
$$

yielding

$$
\Lambda=\frac{W_{1}^{2}}{B_{T} W_{2}-W_{1}^{2}}
$$

The rationale for introducing these auxiliary variables is that the statistical moments of $\Lambda$ cannot be expressed as a direct function of the $\left\{\gamma_{k}\right\}$ and $\left\{f_{k}\right\}$ moments. Nevertheless, the moments of $W_{i}$ can be linked more easily to those of $\left\{\gamma_{k}\right\}$ and $\left\{f_{k}\right\}$; an estimation of the moments of $\Lambda$ can then be given by linearizing (17) around the $W_{i}$ ensemble averages [11]. For the sake of simplicity, the following convention is introduced:

$$
\bar{W}_{i}=\mathrm{E}\left[W_{i} \mid\left\{f_{k}\right\}\right] .
$$

Applying this approach to the average of $\Lambda$ conditional to a given realization $\left\{f_{k}\right\}$ yields

$$
\mathrm{E}\left[\Lambda \mid\left\{f_{k}\right\}\right] \simeq \frac{\bar{W}_{1}^{2}}{B_{T} \bar{W}_{2}-\bar{W}_{1}^{2}}
$$

In the same way, the conditional variance $\sigma_{\Lambda \mid\left\{f_{k}\right\}}^{2}$ can be approximated as

$$
\sigma_{\Lambda \mid\left\{f_{k}\right\}}^{2}=\mathrm{E}\left[\Lambda^{2} \mid\left\{f_{k}\right\}\right]-\mathrm{E}\left[\Lambda \mid\left\{f_{k}\right\}\right]^{2} \simeq \mathbf{J}^{\mathrm{T}} \boldsymbol{\Sigma} \mathbf{J},
$$

where $\boldsymbol{\Sigma}$ is the covariance matrix of the random vector $\left[W_{1}, W_{2}\right]^{\mathrm{T}}$. The column vector $\mathbf{J}$ is the Jacobian of $\Lambda$ as computed with respect to $W_{1}$ and $W_{2}$, evaluated at $\left(\bar{W}_{1}, \bar{W}_{2}\right)$ :

$$
\mathbf{J}=B_{T} \frac{\bar{W}_{1}}{\left(B_{T} \bar{W}_{2}-\bar{W}_{1}^{2}\right)^{2}}\left[\begin{array}{c}
2 \overline{W_{2}} \\
-\overline{W_{1}}
\end{array}\right]
$$

A higher-order estimate of $\Lambda$ could be given, but the resulting expression would be quite unwieldy without delivering considerable improvement in the final accuracy. For the same reason, we just consider the first two moments of $W_{1}$ and $W_{2}$. Thanks to the following expansion

$$
\begin{aligned}
|H(f)|^{2} & =\sum_{k=1}^{M}\left|\gamma_{k}\right|^{2}\left|\phi_{k}(f)\right|^{2}+ \\
& +2 \sum_{k=1}^{M} \sum_{m=k+1}^{M} \operatorname{Re}\left\{\gamma_{k} \gamma_{m}^{\star} \phi_{k}(f) \phi_{m}^{\star}(f)\right\}
\end{aligned}
$$

and assuming the $\left\{\gamma_{k}\right\}$ to be independent from the $\left\{f_{k}\right\}$, while recalling the hypothesis of all the modes having the same en$\operatorname{ergy} \mathcal{E}_{\phi}$, as defined in (5), we can write

$$
\begin{aligned}
\mathrm{E}\left[W_{1} \mid\left\{f_{k}\right\}\right] & =2 M \mu_{2} \mathcal{E}_{\phi} \\
\mathrm{E}\left[W_{2} \mid\left\{f_{k}\right\}\right] & =2 M\left(\mu_{2}^{2}+\mu_{4}\right) \mathcal{E}_{|\phi|^{2}}+ \\
& +16 \mu_{2}^{2} \sum_{k=1}^{M} \sum_{m=k+1}^{M} \mathcal{E}_{\phi_{k} \phi_{m}}
\end{aligned}
$$

having introduced the moments $\mu_{i}$

$$
\mu_{i}=\mathrm{E}\left[\alpha_{k}^{i}\right]=\mathrm{E}\left[\beta_{k}^{i}\right]
$$

Equation (24) differs from (23) in a fundamental aspect, i.e., the presence of the mutual energies $\mathcal{E}_{\phi_{k} \phi_{m}}$ shared between each couple of modes of the system. This term can be shown to be the source of the limitations of the SNR as the modal density $M / B_{T}$ increases enough to lead to nonnegligible interactions between the modes.

The same type of analysis was carried out for the elements $\Sigma_{i j}=\mathrm{E}\left[\left(W_{i}-\mathrm{E}\left[W_{i}\right]\right)\left(W_{j}-\mathrm{E}\left[W_{j}\right]\right)\right]$ of the covariance matrix, but this led to too complex expressions, especially for $\Sigma_{22}$. We would rather propose approximate results, where the mutual-energy terms are neglected:

$$
\boldsymbol{\Sigma} \simeq 4 M \nu,
$$

having defined the elements of $\nu$ as

$$
\begin{aligned}
\nu_{11} & =\mathcal{E}_{\phi}^{2}\left(\mu_{4}-\mu_{2}^{2}\right) \\
\nu_{12} & =\mathcal{E}_{\phi} \mathcal{E}_{|\phi|^{2}}\left(\mu_{2} \mu_{4}+\mu_{6}-2 \mu_{2}^{3}\right) \\
\nu_{22} & =\mathcal{E}_{|\phi|^{2}}^{2}\left(\mu_{8}+4 \mu_{2} \mu_{6}+\mu_{4}^{2}-2 \mu_{2}^{4}-4 \mu_{2}^{2} \mu_{4}\right)
\end{aligned}
$$

It is worth noting that although interactions between modes have been neglected in $\boldsymbol{\Sigma}$, the Jacobian in (21) takes them into account. It will be shown in Section $\mathrm{V}$ that when applied as an input to (20), these expressions provide a good estimate of the variance of $\Lambda$, and as thus they are a useful tool in stating the uncertainty that affects time-reversal performances in a reverberating medium.

\section{AVERAGE ASYMPTOTIC PERFORMANCE}

The formulae presented in the previous Section were derived considering a given deterministic set of resonant frequencies $\left\{f_{k}\right\}$, and as thus (19) and (20) depends, in principle, 
on the actual realization of $\left\{f_{k}\right\}$. In fact, this dependence subsists only in the mutual energies $\mathcal{E}_{\phi_{k} \phi_{m}}$ in (24).

In this Section, we consider the performance of timereversal when averaged over all the realizations of $\left\{f_{k}\right\}$, hence related to its general trend rather than for a specific configuration, proving that under certain conditions, the statistics of a single realization are well approximated by the simpler ensemble statistics.

To this end, let us consider the ensemble average of $\Lambda$ with respect to the random set $\left\{f_{k}\right\}$; thanks to the linearization of $\Lambda$, it will suffice to carry out this averaging over the $W_{i}$, leading to:

$$
\begin{aligned}
\mathrm{E}\left[W_{1}\right] & =2 M \mu_{2} \mathcal{E}_{\phi} \\
\mathrm{E}\left[W_{2}\right] & =2 M\left(\mu_{2}^{2}+\mu_{4}\right) \mathcal{E}_{|\phi|^{2}}+ \\
& +8 \mu_{2}^{2} M(M-1) \mathrm{E}\left[\mathcal{E}_{\phi_{k} \phi_{m}}\right] .
\end{aligned}
$$

The double sum in (24) is thus simplified by introducing the average mutual energy $\mathrm{E}\left[\mathcal{E}_{\phi_{k} \phi_{m}}\right]$. The result in (24) and (31) would then be identical if the following condition were satisfied:

$$
\frac{2}{M(M-1)} \sum_{k=1}^{M} \sum_{m=k+1}^{M} \mathcal{E}_{\phi_{k} \phi_{m}} \simeq \mathrm{E}\left[\mathcal{E}_{\phi_{k} \phi_{m}}\right] .
$$

This requirement corresponds to assuming the system to be ergodic, approximating the ensemble average over all the realizations with the average carried out over the set of mutual energies within a single realization. The strong law of large numbers [12] states that, if the system is ergodic, the left hand of (32) converges in probability to the ensemble average of the mutual energy; therefore, for a sufficiently high number of active modes $M$ one gets

$$
\mathrm{E}\left[\Lambda \mid\left\{f_{k}\right\}\right] \simeq \mathrm{E}[\Lambda]
$$

This phenomenon, often referred to as self-averaging, had already been experimentally highlighted in [13], although in a different context, as one of the most interesting features of time reversal, and it implies that its performance in transmitting coherent pulses in a reverberating medium is asymptotically independent from the actual realization of the set of the resonance frequencies $\left\{f_{k}\right\}$, underpinning the robustness of this technique.

An example highlighting this property is given in Fig. 4, where empirical pdfs are shown for 10 different realizations $\left\{f_{k}\right\}$, for three values of $M$, namely 50, 100 and 200, with $f_{c}=2 \mathrm{GHz}, B_{T}=200 \mathrm{MHz}$ and $Q=1000$. For each realization of $\left\{f_{k}\right\}$, ten thousand sets of modal weights $\left\{\gamma_{k}\right\}$ were considered. It appears that indeed the pdfs converge toward the ensemble average as $M$ increases, even for such small values of $M$.

We can now write

$$
\mathrm{E}[\Lambda]=\frac{2 M \mathcal{E}_{\phi}^{2}}{B_{T}\left[\left(1+\mu_{4} / \mu_{2}^{2}\right) \mathcal{E}_{|\phi|^{2}}+4(M-1) \mathrm{E}\left[\mathcal{E}_{\phi_{k} \phi_{m}}\right]\right]-2 M \mathcal{E}_{\phi}^{2}}
$$

It is clear that the behaviour of $\mathrm{E}[\Lambda]$ could be easily predicted should the three energy terms be known. As a matter of

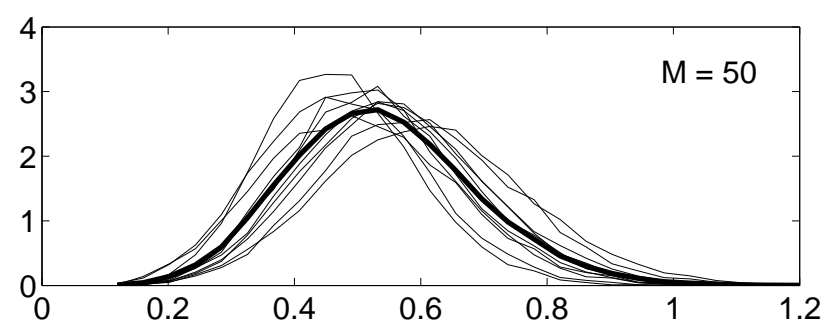

(a)

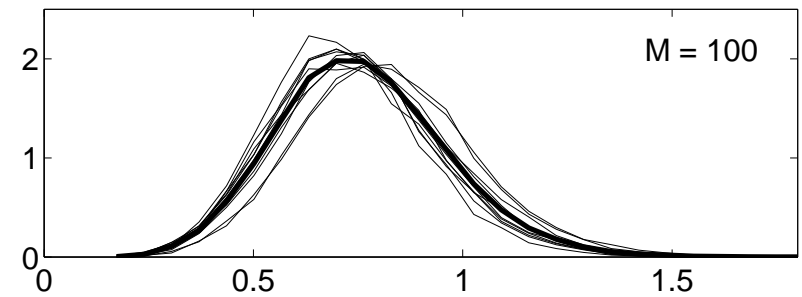

(b)

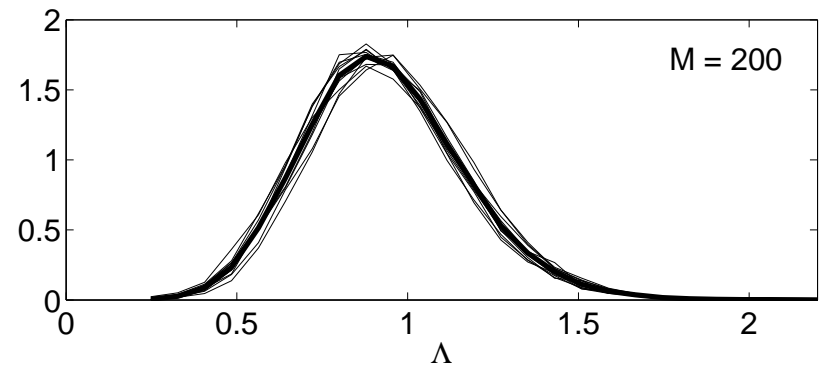

(c)

FIG. 4: Empirical conditional pdfs $p\left(\Lambda \mid\left\{f_{k}\right\}\right)$ for $Q=1000, f_{c}=$ $2 \mathrm{GHz}$ and $B_{T}=200 \mathrm{MHz}$, obtained for an increasing number of active modes. The thicker curve stands for the ensemble average of each group of realizations.

fact, a closed-form expression can be given for $\mathrm{E}\left[\mathcal{E}_{\phi_{k} \phi_{m}}\right]$ by exchanging the order of integration:

$$
\mathrm{E}\left[\mathcal{E}_{\phi_{k} \phi_{m}}\right]=\int_{f_{1}}^{f_{2}}\left(\int_{f_{1}}^{f_{2}}\left|\phi_{k}(f)\right|^{2} p\left(f_{k}\right) \mathrm{d} f_{k}\right)^{2} \mathrm{~d} f
$$

having exploited the fact that the $\left\{f_{k}\right\}$ are iid random variables. Noticing that the inner integral does not depend on the actual frequency $f$ at which it is computed, as long as $B_{T} \gg B_{M}$, (35) becomes

$$
\mathrm{E}\left[\mathcal{E}_{\phi_{k} \phi_{m}}\right]=B_{T}\left(\int_{f_{1}}^{f_{2}}\left|\phi_{k}\left(f_{c}\right)\right|^{2} p\left(f_{k}\right) \mathrm{d} f_{k}\right)^{2}
$$

and by assuming a uniform distribution for the $\left\{f_{k}\right\}$ over the bandwidth $B_{T}$, this yields

$$
\mathrm{E}\left[\mathcal{E}_{\phi_{k} \phi_{m}}\right]=\frac{\mathcal{E}_{\phi}^{2}}{B_{T}}
$$

The two remaining energies $\mathcal{E}_{\phi}$ and $\mathcal{E}_{|\phi|^{2}}$ can also be ex- 
pressed in closed-form as

$$
\begin{aligned}
\mathcal{E}_{\phi_{k}} & =f_{k} \frac{\pi}{2} \frac{Q_{k}}{Q_{k}^{2}+1} \simeq \frac{\pi}{2} B_{M} \\
\mathcal{E}_{\left|\phi_{k}\right|^{2}} & =f_{k} \frac{\pi}{4} \frac{Q_{k}^{3}\left(Q_{k}^{2}+5\right)}{\left(Q_{k}^{2}+1\right)^{3}} \simeq \frac{\pi}{4} B_{M},
\end{aligned}
$$

so that (34) can be restated in a simpler form

$$
\mathrm{E}[\Lambda]=\frac{M}{M+\left(1+\mu_{4} / \mu_{2}^{2}\right) \frac{B_{T}}{2 \pi B_{M}}}
$$

Hence $\mathrm{E}[\Lambda]$ is linearly dependent on $M$ at low modal density levels, whereas it converges to an asymptotic value for an higher $M$. Thus, in lossy reverberating media, the potential gain obtained by increasing the number of active modes (i.e., increasing the central frequency $f_{c}$ ) is put in jeopardy by the coupling existing between lossy resonant modes, with $\mathrm{E}[\Lambda]$ converging to a fixed value. Interestingly, this asymptotic value is simply equal to one. The physical significance of this result will be given a simplified explanation in Section VI.

Equation (40) is remarkably simple, and it shows that a handful of global parameters is sufficient for an accurate prediction of the quality of the received pulse. It is worth noting that the central frequency does not appear explicitly, as a consequence of the identical-mode assumption. These results also point to the fact that the most fundamental quantity for understanding the phenomena behind pulse focusing in a reverberating medium is $B_{T} / B_{M}$. This quantity will be hereafter referred to as $N_{s}$, for reasons that will be made clear in Section VI, yielding

$$
\mathrm{E}[\Lambda]=\frac{M / N_{s}}{M / N_{s}+\left(1+\mu_{4} / \mu_{2}^{2}\right) /(2 \pi)} .
$$

This reformulation states that the average performance is entirely predicted by means of the ratio $M / N_{s}$. As soon as $M \gtrsim N_{s}$ the marginal gain brought by the availability of new modes is increasingly reduced, leading to a saturation for higher $M$.

\section{MODEL VALIDATION}

In order to check the accuracy of the proposed description, we considered numerical simulations, by synthesizing random realizations of transfer functions, thanks to (1). The rationale for this approach is the possibility to closely monitor the number of modes $M$, their quality factor, and so on. Indeed, as recalled later in this Section, experimental validations are impaired by the impossibility to assess the exact number of modes taking part to the transmission.

Thanks to the fact that (40) is not directly dependent on the central frequency $f_{c}$, but rather on the bandwidths $B_{T}$ and $B_{M}$, the validation can be carried out at any value of $f_{c}$. We set for $f_{c}=2 \mathrm{GHz}$, with a varying bandwidth $B_{T}$ and several average quality factors $Q$. Random complex weights $\gamma_{k}$

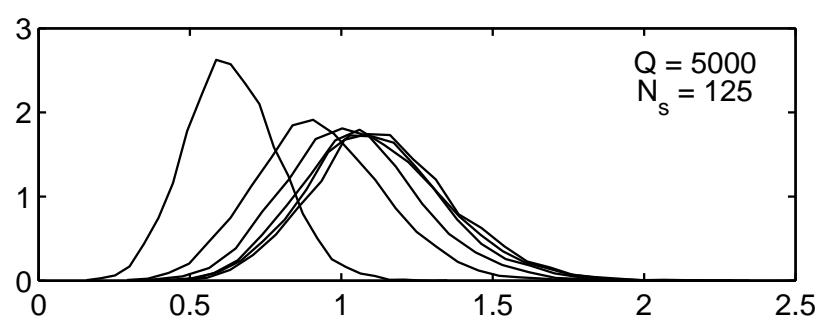

(a)
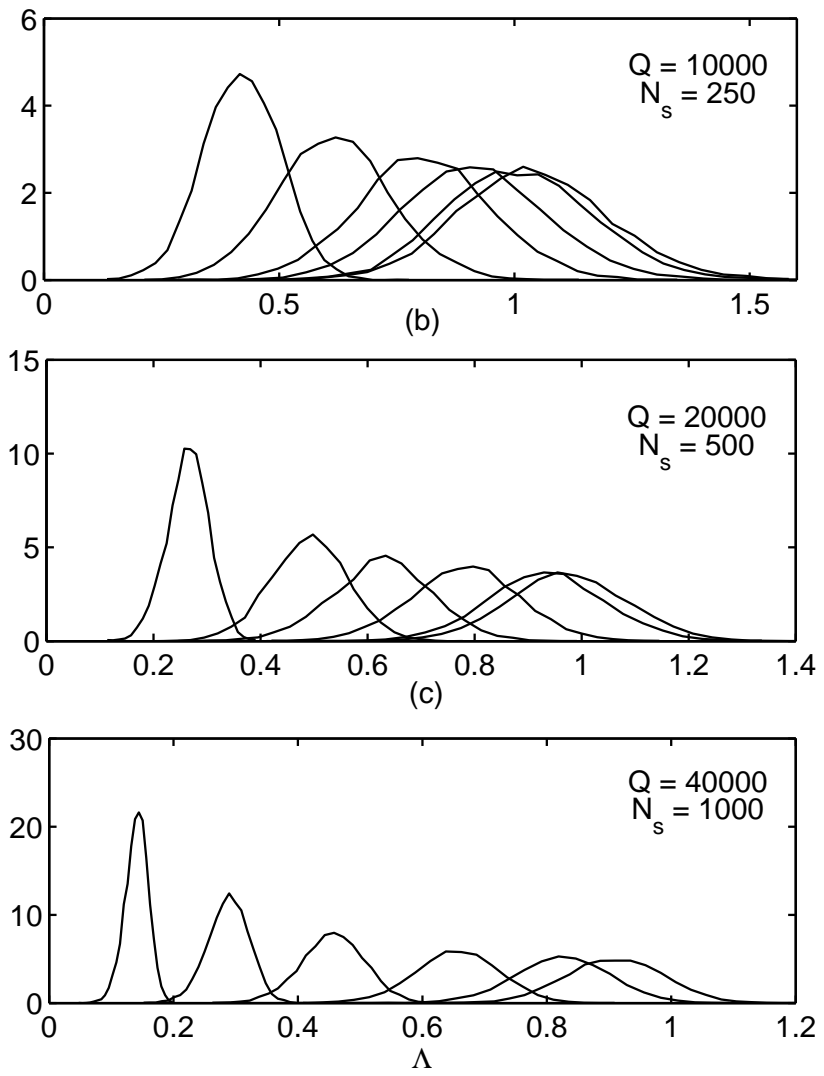

(d)

FIG. 5: Empirical pdfs for the energy SNR $\Lambda$, depending on the number $M$ of active modes and the quality factor $Q$ of the medium. The six curves presented in each picture correspond to $M=\{100,250,500,1000,2500,5000\}$, respectively, from left to right.

are drawn accordingly to normally distributed $\alpha_{k}$ and $\beta_{k}$; the energy SNR for the transfer functions thus obtained are subsequently computed thanks to (8). Contrary to the assumption of a constant $f_{k} / Q_{k}$, the $\phi_{k}(f)$ were assumed to have a constant quality factor $Q=Q_{k}, \forall k$. Therefore, the modal responses $\phi_{k}(f)$ will not be identical as assumed in the model derivation.

The first tests aimed at showing how the energy SNR is distributed as $M$ and $N_{s}=B_{T} / B_{M}$ vary, and is more of a qualitative investigation. A bandwidth $B_{T}$ of $50 \mathrm{MHz}$ was chosen, while four values of $Q$ were tested, ranging from 5000 to 40000 . The number of modes varied from 100 to 5000 . 


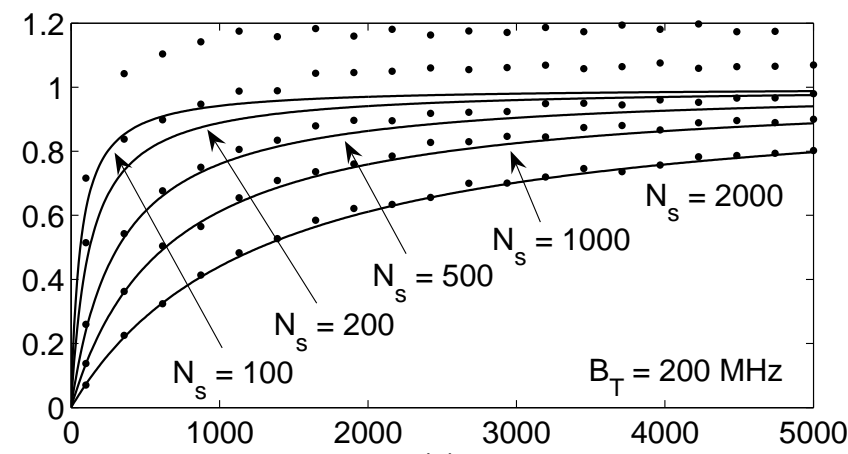

(a)

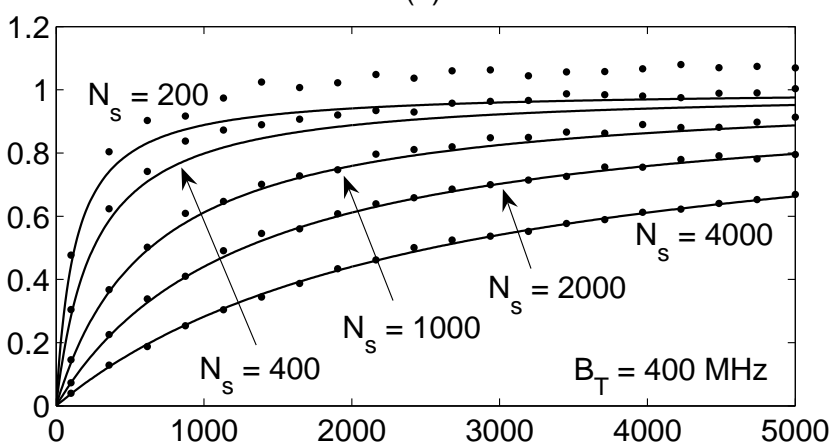

(b)

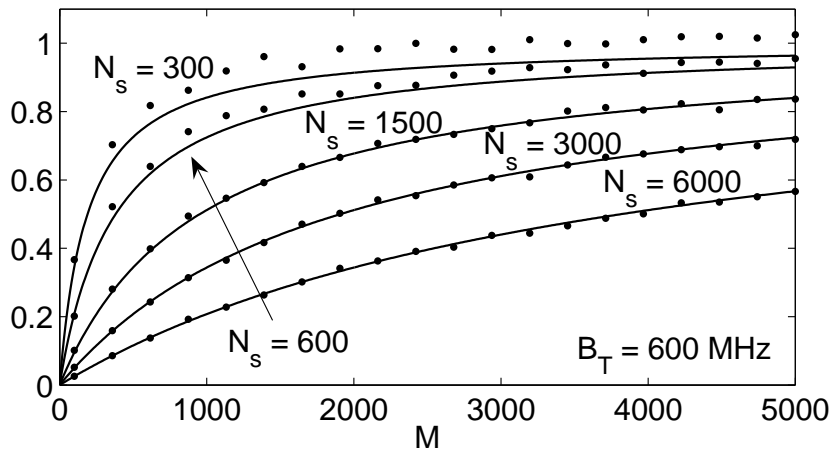

(c)

FIG. 6: Validation tests for $\mathrm{E}[\Lambda]$ : numerical results obtained from 500 realizations (dots) and the values predicted by (40) (solid lines). All the presented results were computed for $f_{c}=2 \mathrm{GHz}$ and for $\mathrm{Q}=\{1000,2000,5000,10000,20000\}$, while the corresponding $N_{s}$ are displayed.

For each set of global parameters ten thousand realizations were generated, in order to establish empirical pdfs; these are shown in Fig. 5, and illustrate quite clearly that: 1) increasing losses tend to saturate the energy SNR faster, as $M$ increases, 2) decreasing losses slow the saturation down, but reduce the average energy SNR, as the length of the residual-noise tails increases and so does the noise energy, and 3) the SNR experiences a standard deviation that is far from negligible when compared to the average value, although, as $Q$ increases, the dispersion appears to decrease. The trend in the simulated pdfs recalls that of the experimental ones shown in Fig. 3.

Even though the energy SNR $\Lambda$ decreases with $Q$, the peak

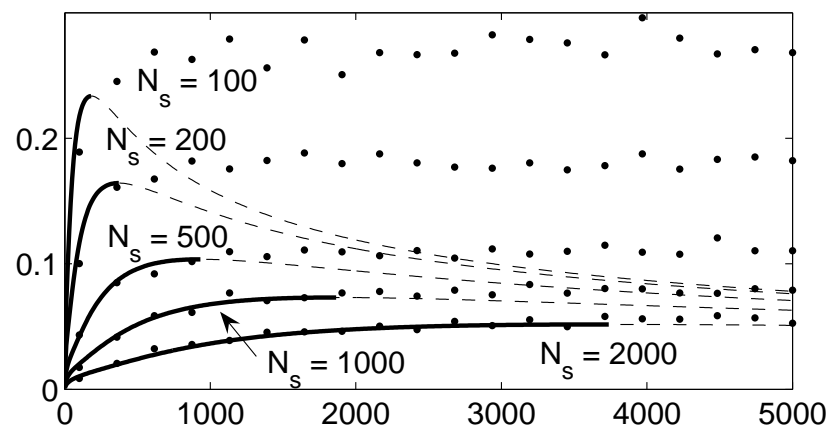

(a)

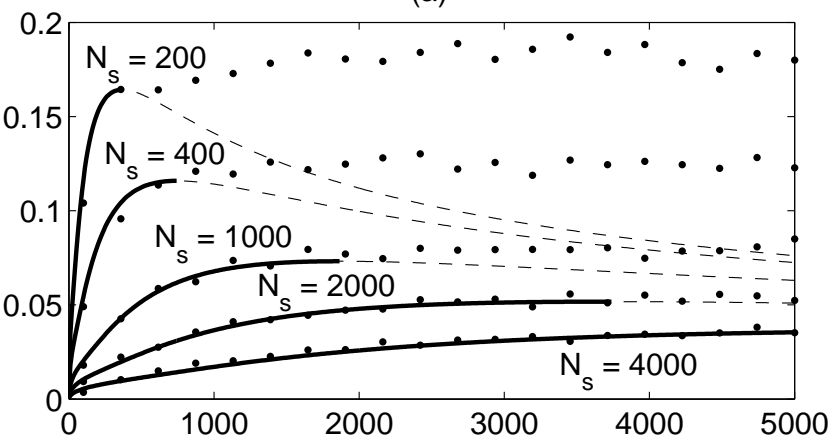

(b)

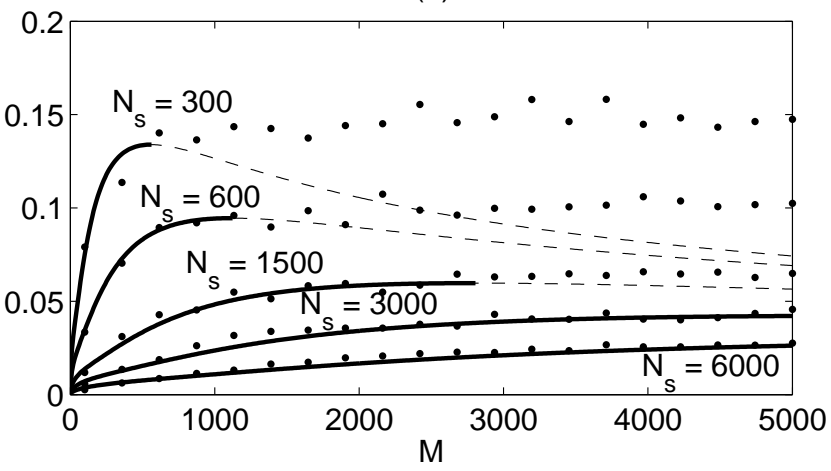

(c)

FIG. 7: Validation tests for the standard deviation of $\Lambda$, for the same configurations as in Fig. 6. The dashed lines correspond to the values predicted by (20), but considering ensemble-averaged $W_{i}$, whereas the thick solid ones highlight the model results up to the curve local maximum, for $M<M_{\sigma}$.

SNR $\Lambda_{p}$ increases monotonically, since the relationship between $\Lambda$ and $Q$ is actually sublinear. This fact is to be expected intuitively, and it also confirms the trend predicted by the model proposed in [4], since

$$
\lim _{Q \rightarrow \infty} \mathrm{E}\left[\Lambda_{p}\right]=M,
$$

again, in the case of a cardinal-sinus pulse and gaussian statistics for the modal weights.

Quantitative validations were then carried out by considering $f_{c}=2 \mathrm{GHz}, Q=\{1000,2500,5000,10000,20000\}$, and a varying bandwidth $B_{T}=\{200,400,600\} \mathrm{MHz}$. The number of modes spanned the values 100 to 5000 , and a population 
of 500 random realizations $\left\{\gamma_{k}\right\}$ per configuration was considered, each configuration representing just a single realization of $\left\{f_{k}\right\}$. The results thus obtained for the average value and the standard deviation of $\Lambda$ are shown in Fig. 6 and Fig. 7, respectively. Fig. 6 shows that the average value is predicted within a few percent points as long as $N_{s} \gtrsim 200$. Indeed, (40) is unable to predict any energy SNR greater than one; this is actually not due to a bad estimate of the moments of the auxiliary variables $W_{i}$, which are indeed precisely estimated in all of the considered tests, since (30) and (31) do not involve any approximation. This rather points directly to the conditions that are necessary for applying the linearization in (9), implying that it is necessary for the condition $N_{s} \gtrsim 200$ to be fulfilled. We investigated the possibility of including the Hessian term in the expansion (19), but we dropped this option, since it brought no tangible improvement, thus implying that the SNR as a non linear function of $W_{i}$ would require terms higher than quadratic ones.

The standard deviation depicts a rather different scenario. Expression (20) neglects any modal interaction in the covariance matrix $\boldsymbol{\Sigma}$, but it includes them through the use of $\bar{W}_{i}$. For this reason, (20) is expected to underperform as soon as the modal interactions get more important, i.e., as the modal density $M / B_{T}$ increases. The results in Fig. 7 support these ideas, showing that (20) is a very good estimate of the standard deviation, as long as it has not yet attained its maximum value $\sigma_{\max }$. After this point, (20) is no more a valid estimate, but the actual standard deviation gets to a plateau fairly approximated by $\sigma_{\max }$. In general, this value needs to be computed numerically, but for the case of modal weights distributed as Gaussian random variables, the number of modes $M_{\sigma}$ for which the standard deviation reaches its maximum value can be approximated by

$$
M_{\sigma} \simeq \frac{6}{\pi} N_{s}
$$

Knowledge of the saturation point allows extending the validity of (20) over the entire range of values of $M$, i.e.,

$$
\sigma_{\Lambda}^{2} \simeq\left\{\begin{array}{c}
\left(\mathbf{J}^{\mathrm{T}} \boldsymbol{\Sigma} \mathbf{J}\right)(M) \quad M \leq M_{\sigma} \\
\frac{1.7 \pi}{N_{s}} \quad M>M_{\sigma}
\end{array} .\right.
$$

Therefore, the maximum standard deviation goes like $1 / \sqrt{N_{s}}$, whereas it is inversely proportional to $N_{s}$ for $M \ll M_{\sigma}$. The former conclusion explains the behaviour previously highlighted, with the standard deviation decreasing when $B_{T}$ and/or $Q$ increase.

These numerical validations prove the effectiveness of the asymptotic models, even for a relatively low number of modes and with no ensemble averaging in $\left\{f_{k}\right\}$. This implies that the ergodic assumption formulated in the previous Section does indeed hold. The greatest limitation in the proposed models is the need for (9) to be well approximated by its tangent plane over the range of values spanned by the $W_{i}$, requiring $N_{s} \gtrsim$ 200.

The last validation is a tentative experimental one. As recalled at the beginning of this Section, the exact number of modes excited in a reverberating system is usually not known.
Therefore a direct validation is not feasible; nevertheless, it is current practice in electrical engineering to assumed that a linearly polarized antenna placed in an electrically large reverberating chamber will excite most of the modes existing over the frequency range of emission of the antenna. As a consequence, Weyl's formula is often used as a reference. Hence, we computed the moments of the energy SNR predicted by our model, considering a number of modes equal to three fractions of the estimate $M_{\mathrm{w}}$ given by Weyl's formula (14).

The results are resumed in Table II, together with the quantity $N_{s}$ : the range of variation of the SNR is very well identified, both for the average value and the standard deviation, and the experimental results are consistently approached when considering a number of modes close to $0.9 M_{\mathrm{w}}$. Furthermore, as expected from the numerical validation, as soon as $N_{s}$ decreases towards 200 the experimental average $\Lambda$ goes beyond one; in this case, the model will underestimate the statistical moments.

The fact that considering the same fraction of $M_{\mathrm{w}}$ over the six frequencies leads to good results, strongly reduces the odds that this accuracy be a random result; we thus consider that $90 \%$ of the available modes were indeed effectively excited. The only exception is for $f_{c}=0.5 \mathrm{GHz}$ and $B_{T}=200 \mathrm{MHz}$; in fact, the transmitting antenna had a cut-off frequency around $450 \mathrm{MHz}$, so that of the $200 \mathrm{MHz}$ pulse to be received, it actually transmitted only three-quarters of the signal spectrum, hence exciting roughly three-quarters of the available modes. By taking into account this fact, the actual number of modes to be considered is rather $3 / 4 \cdot 0.9 M_{\mathrm{w}} \simeq$ $2 / 3 M_{\mathrm{w}}$ : indeed, the results agree.

Overall, it appears that the average of $\Lambda$ is hardly affected by an increase in $B_{T}$. Actually, this is predictable, since both $N_{s}$ and $M_{\mathrm{w}}$ are linearly dependent on $B_{T}$, so that (41) is not modified. Conversely, the peak SNR will increase proportionally to $B_{T}$. At the same time, the standard deviation is sensitive to an increasing $B_{T}$. This was predicted in (44), and the reduction of a factor $1 / \sqrt{2}$ subsequent to a doubling $B_{T}$ is indeed well confirmed by the experimental results. These findings are of the utmost importance should time-reversal techniques be used for pulse transmission.

\section{AN HEURISTIC INTERPRETATION}

We will here try to give an interpretation of the reported phenomena from a more physical, yet approximate, point of view. To this end, let us recall that the maximum value attained by the peak SNR $\Lambda_{p}$, as long as $N_{s} \gtrsim 200$, is simply given by $N_{s} /(2 \pi)$. It is thus not dependent on the actual number of modes $M$, but rather to a, usually, much lower quantity.

Let us look at $N_{s}$ from a different perspective: knowing that $B_{M}$ is the average bandwidth of the frequency response of each mode, $N_{s}$ states the maximum number of modes that could be placed one after the other over the bandwidth $B_{T}$. The energy SNR corresponding to this configuration is equal to one, and it corresponds to the best efficiency time-reversal can provide in concentrating energy in the coherent part rather than in the residual noise. 


\begin{tabular}{cccccccc}
\hline \hline$f_{c}(\mathrm{GHz})$ & $B_{T}(\mathrm{MHz})$ & experiments & mod. $2 M_{\mathrm{w}} / 3$ & $\bmod .3 M_{\mathrm{w}} / 4$ & $\bmod . M_{\mathrm{w}}$ & $N_{s}$ & $M_{\mathrm{w}} / N_{s}$ \\
\hline \multirow{2}{*}{0.5} & 100 & $0.37(0.074)$ & $0.30(0.082)$ & $0.33(0.083)$ & $0.39(0.083)$ & 785 & \multirow{2}{*}{0.41} \\
& 200 & $0.30(0.051)$ & $0.30(0.058)$ & $0.33(0.058)$ & $0.39(0.058)$ & 1570 & \\
\multirow{4}{*}{1.0} & 100 & $0.75(0.12)$ & $0.70(0.097)$ & $0.73(0.097)$ & $0.78(0.097)$ & 570 & \multirow{2}{*}{2.3} \\
& 200 & $0.72(0.080)$ & $0.70(0.069)$ & $0.73(0.069)$ & $0.78(0.069)$ & 1140 & \\
\multirow{3}{*}{1.5} & 100 & $0.91(0.14)$ & $0.88(0.11)$ & $0.89(0.11)$ & $0.92(0.11)$ & 420 & \multirow{2}{*}{6.9} \\
& 200 & $0.90(0.095)$ & $0.88(0.080)$ & $0.89(0.080)$ & $0.92(0.080)$ & 840 & \\
\multirow{2}{*}{2.0} & 100 & $0.96(0.13)$ & $0.95(0.013)$ & $0.95(0.13)$ & $0.96(0.13)$ & 315 & \multirow{2}{*}{16} \\
& 200 & $0.95(0.010)$ & $0.95(0.092)$ & $0.95(0.092)$ & $0.96(0.092)$ & 630 & \\
\multirow{2}{*}{2.5} & 100 & $1.02(0.13)$ & $0.97(0.14)$ & $0.97(0.14)$ & $0.98(0.14)$ & 288 & \multirow{2}{*}{28} \\
& 200 & $1.00(0.12)$ & $0.97(0.097)$ & $0.97(0.097)$ & $0.98(0.097)$ & 576 & \\
\multirow{2}{*}{3.0} & 100 & $1.02(0.14)$ & $0.98(0.14)$ & $0.98(0.14)$ & $0.98(0.14)$ & 290 & \multirow{2}{*}{40} \\
& 200 & $0.97(0.10)$ & $0.98(0.096)$ & $0.98(0.096)$ & $0.98(0.096)$ & 580 & \\
\hline \hline
\end{tabular}

TABLE II: Experimental validation against the results presented in Table I: mean values are given directly, while standard deviations are in parenthesis. The results computed by means of (40) and (20) were obtained considering a number of active modes equal to $2 M_{\mathrm{w}} / 3,3 M_{\mathrm{w}} / 4$ and $M_{\mathrm{w}}$, due to the uncertainty on the actual $M$. The reliability of the estimates can be tested by checking the condition $N_{s} \gtrsim 200$.

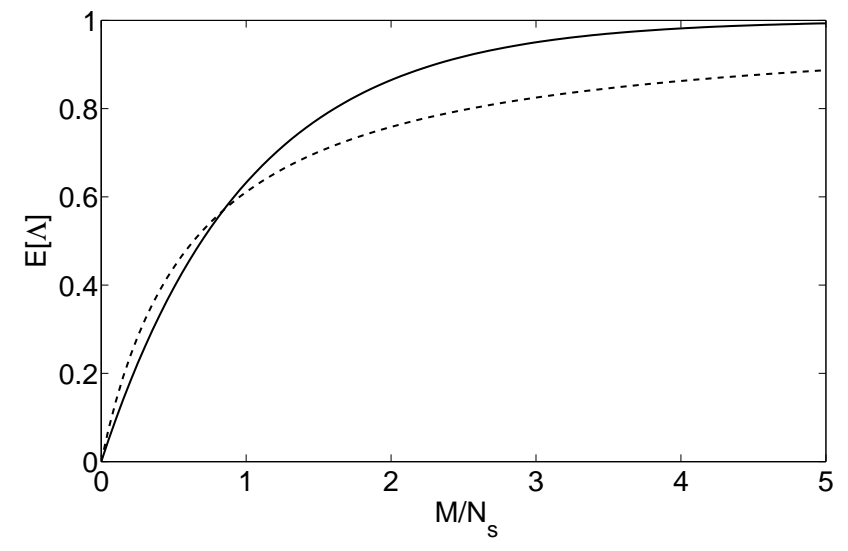

FIG. 8: Comparison between the mean energy SNR, as predicted by the modal approach (41) (solid line) and the slot occupancy description (46) (dashed line).

This fact can be used to give a simple intuitive interpretation, by introducing the idea of a number $N_{s}$ of available slots, to be occupied by the actual number of active modes. Although simplistic, this vision of the spectrum as a quantified space makes sense. Hence, each mode introduces a further degree of freedom only if it can be allocated to a free slot; otherwise, it will be lost, just leading to a different modal weight, as a function depending on the weights of the modes previously allocated to the same slot. Therefore, the performance of time reversal is not related to the actual number of active modes, but rather to the number of slots being used, which could thus be regarded as an effective number of modes or degrees of freedom, or $M_{e}$, leading to an efficiency and, ultimately, to an energy SNR equal to $M_{e} / N_{s}$. The allocation of a mode to a specific slot being a "rare" event, this random process can be modelled by a Poisson law, with mean $M / N_{s}$. The mean number of occupied slots, and thus the effective number of modes $M_{e}$, is thus simply given by

$$
M_{e}=N_{s}\left(1-\mathrm{e}^{-M / N_{s}}\right),
$$

and the related energy SNR

$$
\mathrm{E}[\Lambda] \simeq \frac{M_{e}}{N_{s}}=1-\mathrm{e}^{-M / N_{s}},
$$

highlighting the dominant role of the quantity $M / N_{s}$, as previously shown in (41). We could thus dub the quantity $M / N_{s}$ as the modal slot occupancy: it defines completely the SNR and is sufficient for predicting the performance of time reversal in any configuration.

The validity of this reasoning is proven in Fig. 8. Indeed, for a low number of modes (with respect to $N_{s}$ ) the results predicted by (41) and (46) correspond fairly well. For higher slot occupancies, (46) saturates faster, since this model is incapable of acknowledging the partial superposition of two modes, something that would just lead to a partial loss of a degree of freedom. In spite of this over-simplification, this approach yields results consistent with those predicted by (41), while providing a simple framework for understanding the SNR saturation phenomenon.

\section{CONCLUSIONS}

This paper has addressed the main phenomena underlying the quality of pulses received by a transducer as transmitted through a reverberating medium, when using time-reversal techniques. The quality of the received pulse has been analyzed with respect to global parameters identifying the properties of the medium, according to a modal description. Having included loss mechanisms, it was proven that the sharing of energy between finite-bandwidth resonant modes is at the origin of the limitations in the SNR of the received pulse. A 
statistical approach has led to general results based on very few assumptions, mainly that of a sufficiently "wide-band" configuration with $B_{T} / B_{M} \gtrsim 200$ : the developed model predicts correctly the first two statistical moments of the SNR, acknowledging its non-negligible statistical dispersion. Although mainly based on a mathematical approach, the physical meaning of these results were explained in plain terms by introducing a simplified heuristic description, proving that the SNR is in fact limited by the finite number of degrees of freedom available in a lossy reverberating system. These results should be useful for both the design of experiments and the interpretation of their results, and pertain to any type of wave propagation problem in a reverberating environment.
[1] G. Lerosey, J. De Rosny, A. Tourin, A. Derode, G. Montaldo and M. Fink, Radio Science 40, RS6S12.1 (2005).

[2] L. Borcea, G. Papanicolaou, C. Tsogka and J. Berryman, Inverse Problems 18, 1247 (2002).

[3] C. Prada and M. Fink, Wave Motion 20, 151 (1994).

[4] J. De Rosny, Ph.D. Thesis, Université Paris VI, 2000.

[5] P.M. Morse and H. Feshback, Methods of Theoretical Physics, Feshback Publishing, 1981.

[6] A. Derode, A. Tourin, J. D. Rosny, M. Tanter, S. Yon and M. Fink, Phys. Rev. Lett. 90, 014301-1 (2003).

[7] H. Moussa, A. Cozza and M. Cauterman, IEEE EMC Symposium, Austin, Texas (2009).
[8] C.L. Holloway, D.A. Hill, J.M. Ladbury, P.F. Wilson, G. Koepke and J. Coder, IEEE Trans. on Antennas and Propagation 54, 3167 (2006).

[9] H. Weyl, Math. Ann. 71, 441 (1912).

[10] D.A. Hill, IEEE Trans. on Electromagnetic Compatibility 40, 209 (1998).

[11] A.I. Khuri, Advanced Calculus with Applications in Statistics, Wiley-Interscience, 2002.

[12] A. Leon-Garcia, Probability, Statistics, and Random Processes for Electrical Engineering, Prentice-Hall, 2008.

[13] A. Derode, A. Tourin and M. Fink, Phys. Rev. E 64 (2001). 